Cite this: Phys. Chem. Chem. Phys., 2012, 14, 1881-1891

\title{
On the modeling of aggregates of an optically active regioregular polythiophene
}

\author{
Juan Torras, ${ }^{* a}$ Cristina Sanchez-Navas, ${ }^{a}$ Oscar Bertran $^{b}$ and Carlos Alemán ${ }^{c d}$ \\ Received 3rd October 2011, Accepted 6th December 2011 \\ DOI: $10.1039 / \mathrm{c} 2 \mathrm{cp} 23122 \mathrm{k}$
}

The conformational properties of the optically active regioregular poly[( $R$ )-3-(4-(4-ethyl-2oxazolin-2-yl) phenyl) thiophene] (PEOPT) were explored by molecular dynamics on a single chain using several solvents of increasing polarity. Furthermore, their aggregate formation was studied over a wide range of temperatures using a replica exchange molecular dynamics simulation providing simulation data representative of the equilibrium behaviour of their aggregates. Results show a clear tendency of PEOPT to keep a syn-gauche conformation between continuous backbone thiophene rings favouring a bent chain structure in solvent. After studying their aggregation behaviour in acetonitrile, a strong tendency to pack stabilizing structures that reinforce the chirality of the polymer, in concordance with experimental data, was found. Two different aggregated structures were observed depending on oligomer length, a self-assembled helical aggregate based on stacked octamers and a bent double helix aggregate in large oligomers.

\section{Introduction}

The polythiophene derivatives (PTs) are among the most interesting and useful $\pi$-conjugated polymers due to their interesting electronic and optical properties. ${ }^{1-3}$ Among them, the study of chiral polythiophenes has attracted a special interest due to their unusual chiroptical properties with applications such as in circularly polarized electroluminescence devices and enantioselective sensors. ${ }^{3-5}$ Chiral substituents of PTs allow study of properties of these polymers with circularly polarized light, showing a strong optical activity associated with the $\pi-\pi^{*}$ transition of the PTs main chain. Thus, when the polymer is in an aggregated phase it shows an intense induced circular dichroism (ICD) in the UV-Vis region derived from the main chain and/or supramolecular chirality. However, the environmental conditions have been shown to play an important role in the observed ICD activity because, unlike other optically active polymers, chiral PTs usually exhibit no optical activity in the $\pi-\pi^{*}$ transition region in a good solvent at high temperatures. In contrast, they present unique optical activity in a poor solvent at low temperatures or in the film where

\footnotetext{
a Departament d'Enginyeria Quimica, Escola d'Enginyeria d'Igualada, Universitat Politècnica de Catalunya, Pça. Rei 15, 08700 Igualada,

Spain.E-mail: joan.torras@upc.edu

${ }^{b}$ Departament Física aplicada, Escola d'Enginyeria d'Igualada, Universitat Politècnica de Catalunya, Pça. Rei 15, 08700 Igualada, Spain

${ }^{c}$ Departament d'Enginyeria Quimica, E. T. S. d'Enginyeria Industrial de Barcelona, Universitat Politècnica de Catalunya, Diagonal 647, 08028 Barcelona, Spain

${ }^{d}$ Center for Research in Nano-Engineering, Universitat Politècnica de Catalunya, Campus Sud, Edifici C', C/Pasqual i Vila s/n, Barcelona E-08028, Spain
}

molecular chains tend to aggregate with the ability to form $\pi$-stacked supramolecular structures through intermolecular interactions. ${ }^{6-8}$ Nevertheless, as far as we know, complete structural interpretation of the origin of the optical activity in the aggregated chiral PTs still remains unclear, even though some studies have proposed a helical packing of predominantly planar chains for the chiral aggregated PTs rather than a helical cisoid conformation. ${ }^{9,10}$

Previously, some theoretical studies related to the influence of the solvent on the conformation and aggregation of poly(3-hexylthiophene) (P3HT) had pointed out that two backbones, which are oriented parallel to each other, form a very stable structure. This was attributed to the high attraction energy between the aromatic parts of the molecules, even though it is rather flexible in poor solvents in which the clusters themselves can bend and form a folded structure. ${ }^{11,12}$ In general, self-assembly of $\pi$-conjugated polymers in a dilute solution under limited solubility can afford highly crystalline nanowires. However, under partially soluble conditions, conjugated molecules tend to aggregate in a face-to-face stacking fashion, allowing reduction of unfavourable interaction between the solvent and the aromatic main chain. In fact, with an appropriate choice of solvent, Kiriy and coworkers demonstrated the induction of unique 1D nanocrystalline structures of poly(3-alkylthiophene) (P3AT) with main chain helical conformation. ${ }^{10,13}$ Recently, these solvent-induced $1 \mathrm{D}$ or $2 \mathrm{D}$ aggregates were used as precursors for high-performance organic thin-film transistors made of P3HT.

Addition of acetonitrile to chloroform solvent maximizes the favourable $\pi-\pi^{*}$ stacking interactions in the precursor solution, which improves the molecular ordering of the resulting P3HT thin film and enhances the field-effect mobility without post-treatment. ${ }^{14}$ 


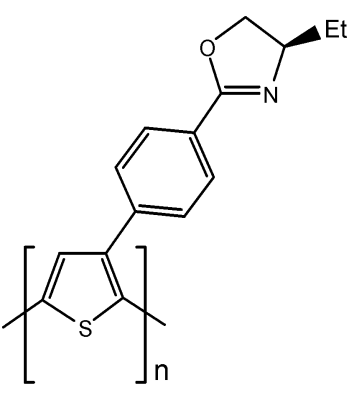

PEOPT

Scheme 1

Among those chiral regioregular PTs, poly[( $R)$-3-(4-(4ethyl-2-oxazolin-2-yl)phenyl)-thiophene] (hereafter PEOPT for the polymer and $n$-EOPT for oligomers with $n$ repeating units; Scheme 1) has been the focus of many studies aimed to show the relationships between the chiroptical properties and their supramolecular structures.

A seminal paper of Yashima et al. ${ }^{4}$ showed that PEOPT chirality could be induced through complexation with metal ions in good solvents, such as chloroform. This feature was attributed to intermolecular coordination of the oxazoline groups to the metal ions, which resulted in the formation of helical arrangements with a well-defined handedness. More specifically, two nitrogen atoms belonging to the non- $\pi$-stacked polymer chains were coordinated with a metal ion. ${ }^{15}$ Moreover, variations in the nature of the solvent induce changes in the ICD spectra. Specifically, the addition of poor solvents to a chloroform solution led to conformational changes that produced chiral aggregations. ${ }^{5}$ Interestingly, a size dependence to form aggregates was observed in the short chain $n$-EOPT oligomers, where solvent-induced Cotton effects were only detected for $n=8$ (i.e. no induced $\mathrm{CD}$ was found in smaller oligomers) ${ }^{16}$ Interestingly, a chirality switching process for such PEOPT aggregates was conducted by the alternative addition of a dicationic metal and the metal complexing agent, leading to back and forth aggregate formation. ${ }^{17}$

Several theoretical studies of chiral regioregular PTs devoted to connect the solvent-induced and the thermochromic conformational transitions with the torsional potential, dispersion of dihedral angles between thiophene rings, and the mechanism of flexibility of the PT chain have been carried out. ${ }^{11,12}$ Recently, a comprehensive theoretical study on $n$-EOPT oligomers, based on the results provided by an exhaustive conformational study on oligomers, proposed a planar ring structure and a helical conformation for the 8-EOPT and PEOPT polymers, respectively. ${ }^{18}$ In general, the problem of formation of different chiral structures from the chiral monomeric units remains a challenge from both theoretical and experimental points of view. The aim of the present paper is to study by means of Molecular Dynamics (MD) and Replica Exchange Molecular Dynamics (REMD) ${ }^{19,20}$ simulations the behaviour of single polymer chains and small aggregates of PEOPT in poor and good solvents. Accordingly, the paper is organized as follows. Initially, the solvent effect on the strained, bent, and helical conformations of the PEOPT is studied by analysing the conformational dispersion of the inter-ring dihedral angle between consecutive thiophene rings, considering different solvent polarities. Secondly, a short study of PEOPT chiral aggregation between two short chains is carried out using REMD methodology. Finally, the chiral aggregates formation, starting from 8-EOPT as building blocks, in the presence of acetonitrile has been addressed by studying the tertiary structure of a set of oligomers made of $16 \pi$-stacked octamers, using REMD methodology.

\section{Methods}

\section{Simulated systems}

The sequences for the five conducting oligomers and aggregates investigated in this work, hereafter referred as $\mathrm{I}-\mathrm{V}$, are listed in Table 1. These structures were built following a previous work, ${ }^{18}$ in which the head-tail $(\mathrm{H}-\mathrm{T})$ isomer was identified as the most stable. Specifically, two minima were identified for the $\mathrm{H}-\mathrm{T}$ 2-EOPT (i.e. $s g$ and $a g$ with the inter-ring dihedral angle located at $51.7^{\circ}$ and $120.2^{\circ}$, respectively), and the three maxima were located with the inter-ring dihedral angle arrangements of syn, gauche-gauche, and anti (i.e. about $0^{\circ}, 100^{\circ}$, and $180^{\circ}$, respectively). In this work two secondary structures were considered as starting points for the polymeric models. The two structures, which were constructed considering 20 repeating units arranged according to an $\mathrm{H}-\mathrm{T}$ disposition, consisted of the all-sg and all-ag conformations, the initial inter-ring dihedral angles being $60^{\circ}$ and $120^{\circ}$, respectively. The force field parameter of inter-ring dihedral angle $\mathrm{S}-\mathrm{C}-\mathrm{C}-\mathrm{S}$ between consecutive thiophene rings was derived from a previous work ${ }^{21}$ while the rest of the force field parameters used in the MD and REMD simulations were extrapolated from the Generalized AMBER Force Field (GAFF) ${ }^{22}$ All trajectories were carried out using the AMBER $11^{23}$ software package. Simulations were performed in chloroform, methanol, and acetonitrile solutions using explicit solvent molecules for models labelled as I and II in Table 1. Polymer molecules were solvated in an orthorhombic simulation box with enough solvent molecules to allow a $10 \AA$ buffer region around the solute. Specifically, the number of chloroform, methanol, and acetonitrile solvent molecules contained in the simulation box was 4642, 3284, and 9473 for the all-sg helix (model I), and 2368, 2088, and 4979 for the all-ag conformation (model II), respectively. All the solvent models used within the first part of this work (i.e. chloroform, ${ }^{24}$ methanol, ${ }^{24}$ and acetonitrile ${ }^{25}$ ) were all atom and compatible with the AMBER force-field employed in all simulations.

The second part of this work studies the aggregation of two oligomers in acetonitrile solution using implicit solvent. The two oligomers were initially arranged in two different ways. The first one is made of two chains with an extended conformation (all- $a g)$ disposed in parallel to each other: $\left(20-\mathrm{EOPT}_{\text {all- } a g}\right)_{2}$ (model III in Table 1). The second arrangement consists of two chains with a wide helical shape conformation (all-sg) with the molecular backbones intertwined parallel to each other like a double helix but stacked by thiophene rings: $\left(20-\mathrm{EOPT}_{\text {all-sg }}\right)_{2}$ (model IV in Table 1).

Finally, the last part of this work is devoted to study the tertiary structure of an aggregate made of short oligomers 
Table 1 Label, chemical formula and schematic picture of the PEOPT polymers and aggregates studied in this work. The schematic pictures specified the axial, equatorial, and detailed side chain projections of the structures used as starting point in simulations

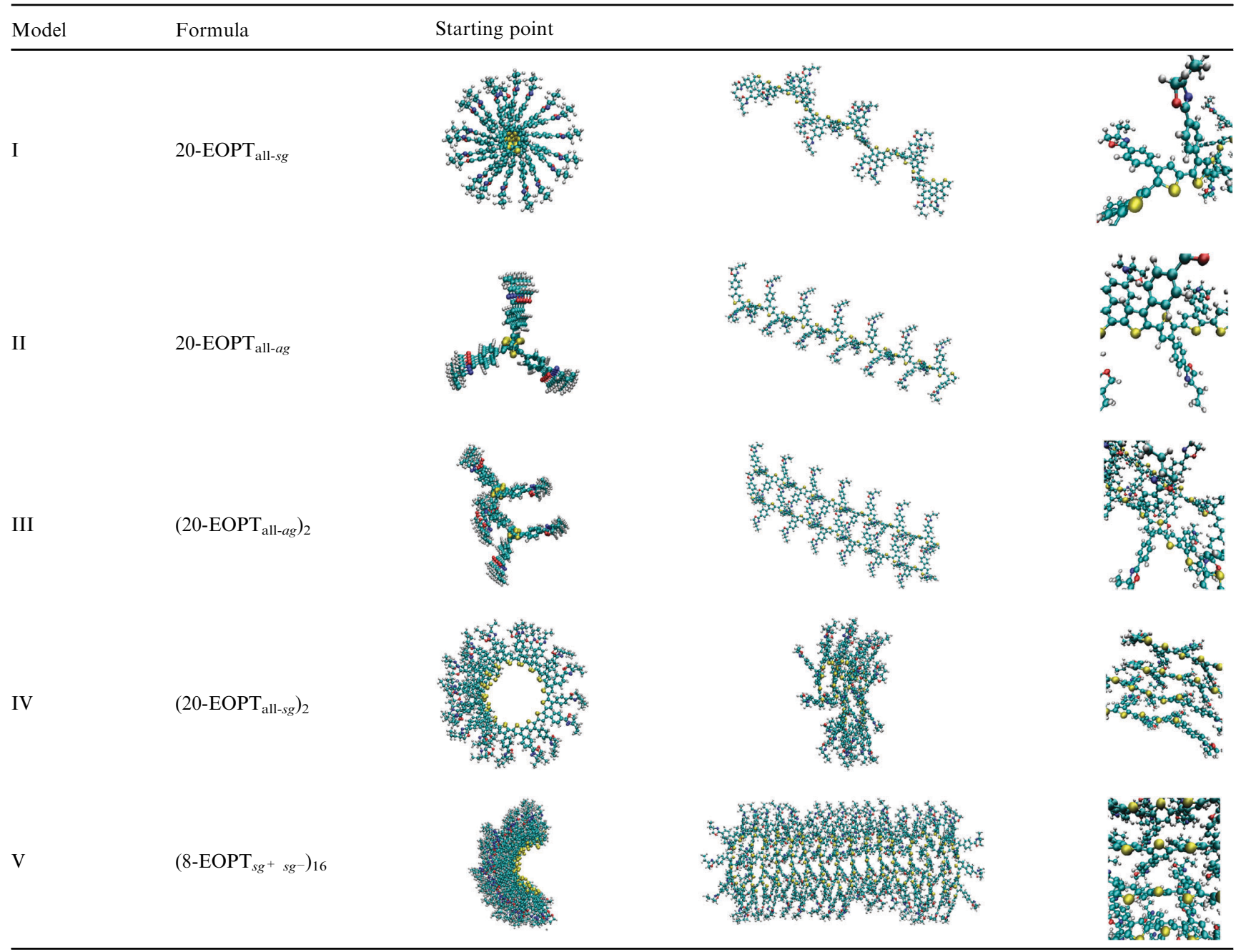

$\left(8-\mathrm{EOPT}_{s g^{+}} s g^{-}\right)$in acetonitrile solution using implicit solvent. Thus, according to the most stable conformation proposed for the 8-EOPT octamer, ${ }^{18}$ which consists of a planar semi-circle, an initial aggregate was built by grouping $16 \pi$-stacked oligomers. This yielded a semitubular structure: $\left(8-\mathrm{EOPT}_{s g^{+}} s g^{-}\right)_{16}$ (model $\mathrm{V}$ in Table 1).

\section{Molecular dynamics simulations}

Classical atomistic MD simulations were performed on models I and II using explicit solvent and periodic boundary conditions. The system preparation has been the same for the two models. Before the production series, every system was equilibrated under the experimental conditions and to the investigation requirements. Thus, for each polymer-solvent pair the main chain conformation was kept close to the starting structure using quadratic harmonic restraints with a constant force of $20 \mathrm{kcal} \mathrm{mol}^{-1}$ over all the solute atoms. Each system was subjected to $3.5 \times 10^{4}$ minimization steps, using the MM Hamiltonian to remove any atomic contact, and then heated to $298 \mathrm{~K}$ for $50 \mathrm{ps}$ at constant volume. The numerical integration step was set to $2.0 \mathrm{fs}$, and the Langevin thermostat was used with a $1.0 \mathrm{ps}^{-1}$ collision frequency. Finally, the system was allowed to relax for an additional 500 ps of isobaric-isothermal relaxation using a NPT ensemble at $298 \mathrm{~K}$ and 1 atmosphere of pressure. All of these steps were performed using the SHAKE algorithm ${ }^{26}$ to keep the bond lengths involving hydrogen atoms at their equilibrium distance. Atom pair distance cut-offs were applied at $10.0 \AA$ to compute the van der Waals interactions. Electrostatic interactions were extensively computed by means of Ewald summations. ${ }^{27}$ Finally, the harmonic restraints that restrict the positions of the solute atoms were released and the production series were obtained under the same conditions previously described for $30 \mathrm{~ns}$. The coordinates of production trajectories were saved every 5000 steps (5 ps intervals) for subsequent analysis.

\section{Replica exchange molecular dynamics}

Models III, IV and V, which correspond to aggregates (see Table 1), have been simulated using the generalized Born (GB) implicit solvent model ${ }^{28}$ of acetonitrile. All simulations were performed using the REMD ${ }^{19,20}$ methodology implemented in the AMBER 11 package. ${ }^{23}$ For models III and IV (1422 atoms) a total number of 32 replicas were exponentially distributed in the temperature from $281.9 \mathrm{~K}$ to $741.2 \mathrm{~K}$, while 16 replicas 
from $292.7 \mathrm{~K}$ to $422.7 \mathrm{~K}$ were considered for model V (4000 atoms). REMD trajectories were carried out using an attempt to exchange every 0.4 ps between all neighbouring replicas with final average acceptance rates of $35 \%$ (models III and IV) and $20 \%$ (model V). Trajectories resulted in a cumulative simulation time of 26 (models III and IV) and $20 \mathrm{~ns}$ (model V). Simulations were run using the SHAKE algorithm ${ }^{26}$ to constrain all the bonds involving hydrogen atoms, with an integration step of 2 fs and an infinite nonbonded cut-off. The replicas were previously equilibrated by a set of short runs (isothermal equilibration) of $5 \mathrm{ps}$ to ensure that each replica achieved the target temperature using a Langevin thermostat with a friction coefficient of $2.5 \mathrm{ps}^{-1}$.

The REMD data were collected from the last 4 ns of simulation at the lowest targeted temperature of 281.9 (32 replicas for models III and IV) and $292.7 \mathrm{~K}$ (16 replicas for model $\mathrm{V}$ ) and at the common targeted temperature of $300 \mathrm{~K}$ to perform data analyses. In order to evaluate the aggregation shape and conformation during REMD simulations, the following parameters were calculated:

- The radius of gyration $\left(R_{\mathrm{g}}, \AA\right)$, which is defined as the root mean square distance of the collection of atoms from their common centre of mass.

- The solvent accessible surface area (SASA, $\mathrm{nm}^{2}$ ), ${ }^{29,30}$ which is computed numerically by representing each atomic surface by spherically distributed dots.

- The shape parameters $K_{1}$ and $K_{2}$ of Khalatur $^{31,32}$ to distinguish between coil and globule shapes of aggregates: $\left(K_{1}=\left(L_{2}+L_{3}\right) /\left(L_{1}+L_{2}\right)\right.$ and $K_{2}=\left(L_{1}+L_{3}\right) /\left(L_{1}+L_{2}\right)$, where $L_{1}, L_{2}$, and $L_{3}$ are the three principal moments of inertia of the gyration tensor of aggregates).

- A clustering analysis to group structures based on root-mean-square deviation (RMSD) as similarity metric. ${ }^{33}$

The post processing of all trajectories was carried out using the ptraj program from the AMBER $11^{23}$ package.

\section{Results and discussion}

\section{Polymer's structure}

A set of several MD simulations were performed to determine the role played by the inter-ring dihedral angle $\theta$ in the PEOPT backbone structure (Scheme 2). Hence, different starting points, which consist of the helical conformation (all-sg, model I) and extended conformation (all-ag, model II), were considered within solvents of growing polarity. Fig. 1 plots the temporal evolution of the root-mean square deviation (RMSD) of the backbone atoms calculated with respect to model I (Fig. 1a) and model II (Fig. 1b). As can be seen, after 20 ns of simulation,

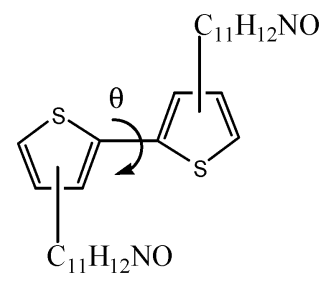

Scheme 2

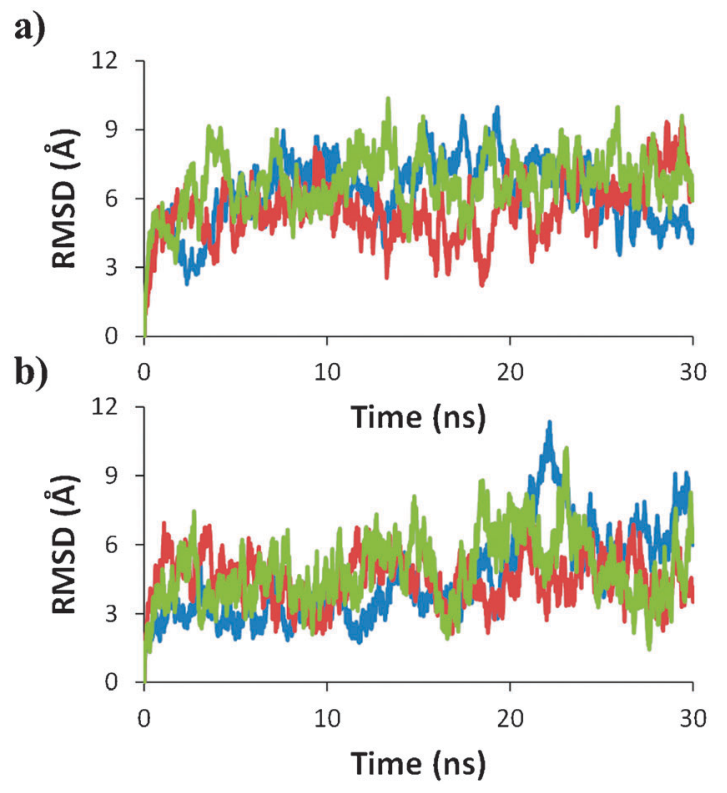

Fig. 1 Time dependent RMSD evolution of the simulations performed with models I (a) and II (b) in chloroform (blue line), methanol (red line), and acetonitrile (green line).

all the systems preserve their structures, i.e. with an RMSD standard deviation within $1 \AA$.

Table 2 lists the population of the inter-ring dihedral angle $\theta$ in the PEOPT backbone structure with a syn-gauche conformation $\left(\mathrm{Pop}_{\theta}^{s g}\right)$. There is a clear tendency to keep the syn-gauche conformation for all the simulated solvents. More specifically, $\mathrm{Pop}_{\theta}^{s g}$ remains almost invariable $(\sim 88-93 \%)$ for systems with an initial all-sg conformation (model I), whereas systems with an initial all-ag structure show an important increase in the syn-gauche dihedral angle population $(\sim 35-42 \%)$. These features indicate a tendency to stabilize a growing number of syn-gauche rotamers. At this point the polarity of the environment does not play an important role in the stabilization of syn-gauche conformation. In fact, the influence of the solvent on $\mathrm{Pop}_{\theta}^{s g}$ is practically negligible.

Regarding the averaged values of inter-ring dihedral angles disposed in a syn-gauche conformation $\left(\theta_{s g}\right.$ in Table 2$)$, they show small differences. Specifically, a difference of about two degrees was observed between the trajectories that started using the all-sg $\left(\theta_{s g} \approx 41^{\circ}\right)$ and all-ag $\left(\theta_{s g} \approx 39^{\circ}\right)$ conformations. Similarly, comparison of averaged values of the inter-ring dihedral angle disposed in anti-gauche ( $\theta_{a g}$ in Table 2$)$ is very small: $\theta_{a g} \approx 140^{\circ}$ and $\sim 138^{\circ}$ for trajectories obtained using the all-ag and all-sg, respectively, as starting conformation. Comparison of the averages obtained in these simulations with the most stable conformations of 2-EOPT dimers obtained in a previous work ${ }^{34}$ reveals that the dihedral angle obtained in the current study for the syn-gauche conformation $\left(\theta_{s g} \approx 41^{\circ}\right)$ is lower than that predicted by quantum mechanical calculations $\left(\theta_{s g}=51.7^{\circ}\right)$. In contrast, the dihedral angle predicted by quantum mechanical calculations for the anti-gauche conformation $\left(\theta_{a g}=120.2^{\circ}\right)$ is lower than that obtained in this work using MD simulations $\left(\theta_{a g} \approx 139^{\circ}\right)$. These differences can be attributed to the stress induced by the interactions between the side groups and the backbone on the conformation. 
Table 2 Population of inter-ring dihedral angles disposed in the syn-gauche conformation (Pop s $_{\theta}^{\text {sg }}$, averaged values of inter-ring dihedral angles disposed in syn-gauche $\left(\theta_{s g}\right)$ and anti-gauche $\left(\theta_{a g}\right)$, end-to-end distance $\left(d_{\mathrm{e}-\mathrm{e}}\right)$, shape parameters $\left(K_{1}\right.$ and $\left.K_{2}\right)$, and density $(\rho)$, derived from MD simulations of models I (all-sg) and II (all- $a g$ ) in chloroform, methanol, and acetonitrile solutions. Standard deviations are indicated in all averages

\begin{tabular}{|c|c|c|c|c|c|c|}
\hline \multirow[b]{2}{*}{ Model } & \multicolumn{2}{|l|}{ Chloroform } & \multicolumn{2}{|l|}{ Methanol } & \multicolumn{2}{|l|}{ Acetonitrile } \\
\hline & I & II & I & II & I & II \\
\hline $\mathrm{Pop}_{\theta}^{s g}(\%)$ & 93.0 & 41.9 & 88.9 & 39.7 & 93.2 & 35.3 \\
\hline$\theta_{s g} /^{\circ}$ & $41.0 \pm 9.3$ & $38.7 \pm 9.4$ & $40.5 \pm 9.1$ & $38.5 \pm 9.1$ & $41.4 \pm 9.4$ & $38.5 \pm 9.2$ \\
\hline$\left.\theta_{a g}\right|^{\circ}$ & $139.5 \pm 9.4$ & $140.7 \pm 8.9$ & $138.4 \pm 8.9$ & $140.3 \pm 8.5$ & $138.1 \pm 9.3$ & $139.5 \pm 8.9$ \\
\hline$d_{\mathrm{e}-\mathrm{e}} / \AA$ & $42 \pm 5$ & $49 \pm 7$ & $43 \pm 4$ & $46 \pm 7$ & $42 \pm 7$ & $33 \pm 7$ \\
\hline$K_{1}$ & $0,47 \pm 0.11$ & $0,14 \pm 0.06$ & $0,44 \pm 0.09$ & $0,13 \pm 0.02$ & $0,36 \pm 0.07$ & $0,15 \pm 0.04$ \\
\hline$K_{2}$ & $0.92 \pm 0.04$ & $0.95 \pm 0.04$ & $0.91 \pm 0.03$ & $0.94 \pm 0.02$ & $0.91 \pm 0.03$ & $0.95 \pm 0.03$ \\
\hline$\rho / \mathrm{g} \mathrm{L}^{-1}$ & $1.465 \pm 0.002$ & $1.465 \pm 0.003$ & $0.814 \pm 0.001$ & $0.819 \pm 0.001$ & $0.779 \pm 0.002$ & $0.786 \pm 0.002$ \\
\hline
\end{tabular}

In order to ascertain the tendency of PEOPT to hold an extended or bent structure, the variation of the end-to-end distance $\left(d_{\mathrm{e}-\mathrm{e}}\right)$ was investigated. This parameter was defined as the distance between the first and last sulfur atoms. Table 2 includes the average values of $d_{\mathrm{e} e}$ for the different models and solvents. For model I, the resulting averaged values $\left(d_{\mathrm{e}-\mathrm{e}} \approx 42 \AA\right)$, which were very similar in all cases, reflect a significant elongation with respect to the value obtained after minimization of all-sg conformation $\left(d_{\mathrm{e}-\mathrm{e}}=27 \AA\right)$. Moreover, we observe that in all three solvents the distance increases rapidly at the beginning of the simulation, showing a clear evolution from the initial compact helix to a more elongated and stable conformation. On the other hand, the solvent has a significant influence on the end-to-end distance of model II. More specifically, the value obtained in acetonitrile $\left(d_{\mathrm{e}-\mathrm{e}} \approx 33 \AA\right)$ is much smaller than those obtained in methanol and chloroform $\left(d_{\mathrm{e}-\mathrm{e}} \approx\right.$ 46-49 A). Thus, the polymer adopts a folded conformation in acetonitrile while in a less polar environment a less compact conformation was obtained. However, in all cases the conformation was considerably more folded than the initial all-ag conformation $\left(d_{\mathrm{e}-\mathrm{e}}=72 \AA\right)$. A close inspection of trajectory structures indicates that these folded and compact conformations are the consequence of bending distortions on the polymer backbone. Considering that a rod shape is obtained when $K_{1}$ and $K_{2}$ tend to 0 and 1 , respectively, the shape parameters do not show much difference between solvents but their starting point structure. However, a glance at the $K_{1}$ shape parameter shows a little tendency to decrease as solvent polarity increase is observed on model I, approaching a rod shape.

In order to study the interactions involving the side groups during simulation of different systems, radial distribution functions

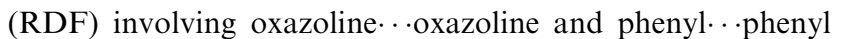
interactions ( $g_{\mathrm{Ox}}(r)$ and $g_{\mathrm{Ph}}(r)$, respectively) were derived from trajectories of models I and II. Thus, the centre of mass of the five-membered ring of oxazoline and the centre of mass of the six-membered ring of a phenyl moiety were considered to obtain their respective RDF. Table 3 lists the RDF maxima regarding oxazoline and phenyl moieties for the three solvents used in this work, while Fig. 2 displays the accumulated $g_{\mathrm{Ox}}(r)$ and $g_{\mathrm{Ph}}(r)$.

The interactions between the different side groups are evidenced by observing the distances of maximum probability between them (Table 3). Systems with a greater number of syn-gauche rotamers (model I) show always a closer interaction distance than those which have a greater number of anti-gauche rotamers (model II) in both $g_{\mathrm{Ox}}(r)$ and $g_{\mathrm{Ph}}(r)$. It is interesting to
Table 3 Position of peaks maxima (in $\AA$ ) in the oxazoline . . oxazoline and phenyl $\cdots$ phenyl radial distribution functions $\left(g_{\mathrm{Ox}}(r)\right.$ and $g_{\mathrm{Ph}}(r)$ respectively) derived from MD simulations of models I (all-sg) and II (all- $a g$ ) in chloroform, methanol, and acetonitrile solutions. See Fig. 1

\begin{tabular}{llll}
\hline Model & Chloroform & Methanol & Acetonitrile \\
\hline I & 8.54 & $g_{\mathrm{Ox}}(r)$ & \\
$\mathrm{II}$ & 9.53 & 8.47 & 8.40 \\
& & 9.70 & 9.66 \\
$\mathrm{I}^{a}$ & $6.10-8.71$ & $g_{\mathrm{Ph}}(r)$ & \\
$\mathrm{II}^{a}$ & $6.33-8.65$ & $6.07-8.67$ & $6.06-8.68$ \\
${ }^{a}$ First and second maxima from $g_{\mathrm{Ph}}(r)$. & $6.24-8.64$ \\
\hline
\end{tabular}

note the behaviours of side group interactions by increasing solvent polarity. In all cases, maxima positions in different solvents are located very close among them, even though a slight tendency to shorten when the solvent polarity increase is detected. Thus, a more polar environment seems to favour slightly the interactions between aromatic fractions of the repeating unit side group. The average distance between the first peaks of an oxazoline moiety in $g_{\mathrm{Ox}}(r)$ presents an appreciable difference depending on the origin of the simulated system. In fact, the oxazoline moiety is located $\sim 1.2 \AA$ farther apart on model II than on model I. However, in the case of the phenyl moiety the difference on model II is only $\sim 0.2 \AA$ away from model I, the first most probable values between the centres of mass of the phenyl groups being at distances of 6.1-6.3 $\AA$. These values are close to the previously derived theoretical distance between phenyl centroids of adjacent repeating units on the syn-gauche conformation for the $\mathrm{H}-\mathrm{T}$ isomer of 2-EOPT, $d_{\mathrm{phs}}=6.69 \AA \AA^{18}$ Similarly, we found that the second set of maxima peaks from $g_{\mathrm{Ph}}(r)$, with values ranging from 8.6 to $8.7 \AA$, is close to that predicted for the anti-gauche conformation for the H-T isomer of 2-EOPT, $d_{\mathrm{phs}}=7.98 \AA .^{18}$

Fig. $2 \mathrm{a}$ and $\mathrm{b}$ show how the interaction between oxazoline moieties is more favoured in model I than in model II, the probability of the first peak of $g_{\mathrm{Ox}}(r)$ RDF being much lower in the latter than in the former. It is also noted that acetonitrile solvent clearly favours the interaction between oxazoline moieties of model I. In Fig. 2c and d, the main difference corresponds to the intensity of the first and second $g_{\mathrm{Ph}}(r)$ peaks, which shows a strong dependence on the population of syn-gauche and anti-gauche rotamers for the inter-ring dihedral angles. Indeed, model I, with a high population of syn-gauche rotamers, presents the greatest intensity of the first $g_{\mathrm{Ph}}(r)$ peak, as shown in Fig. 2a. On the other hand, model II, with similar 


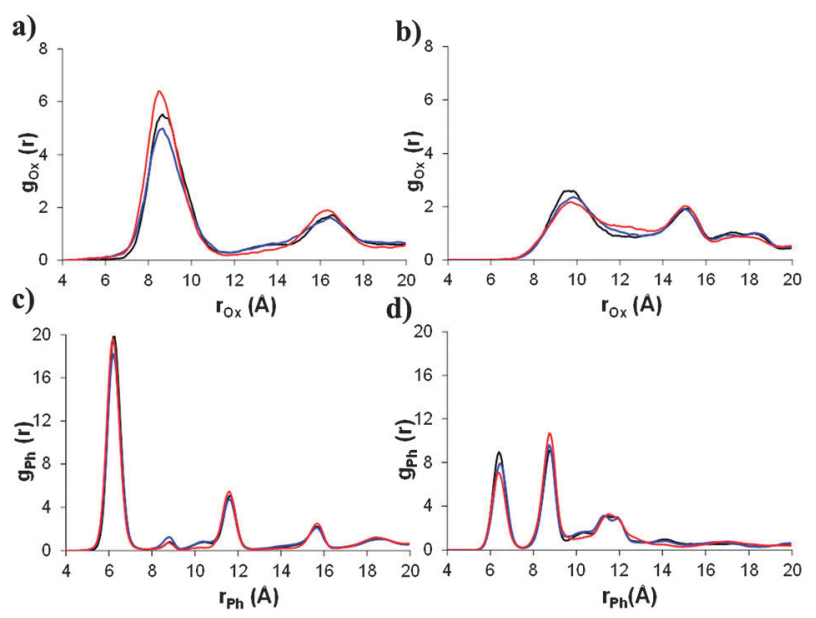

Fig. 2 Oxazoline $\cdots$ oxazoline radial distribution functions, $g_{\mathrm{Ox}}(r)$, derived from MD simulations of (a) model I (all-sg), and (b) model II

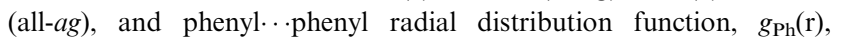
derived from MD simulations of (c) model I (all-sg), and (d) model II (all-ag) in chloroform (black line), methanol (blue line), and acetonitrile (red line) solutions.

populations for both anti-gauche and syn-gauche rotamers, displays similar probability values for the first two peaks of $g_{\mathrm{Ph}}(r)$ (Fig. 2d).

\section{Oligomer aggregation}

Studies of aggregates formation were carried out using the REMD methodology with a minimal system made of two chains of twenty monomeric units. Two models, showing initial extended (model III) and helical (model IV) conformations, were considered (Table 1). Since in the previous section the bent conformation was shown to be the most favoured in polar solvents, these two very different starting points allow us to determine the preferred conformation and its stabilities. Therefore, aggregation effects regarding shape stabilization will also be studied.

Fig. 3 shows the time evolutions of folding indicators such as $R_{\mathrm{g}}$ and SASA, which were extracted from $26 \mathrm{~ns}$ of REMD trajectory at $300 \mathrm{~K}$. This figure displays the greatest stability of the helical conformation regarding the extended one which is reflected by the slow decreases in both $R_{\mathrm{g}}$ and SASA values. On the other hand, the extended conformation quickly collapses to a more globular shape explaining the dramatic decrease in $R_{\mathrm{g}}$, which evolves asymptotically towards the same value as that for the helical conformation. Moreover, time evolution of SASA values presents the same trend, although this is not as pronounced as the first. In fact, the observed decrease in the SASA corresponds to burying of the hydrophobic moieties within the aggregate, favouring aromatic interactions (e.g. those between thiophene and phenyl moieties). However, this reorganization is not as large as that expected on a helical conformation, indicating a globular structure instead, whose shape will be studied below.

The contour maps, indicating the population of the studied aggregates, are plotted against a 2D space (Fig. 4 and 5). Two different contour maps are shown: Fig. 4 corresponds to the $2 \mathrm{D}$ space $R_{\mathrm{g}} v s$. potential energy, whereas Fig. 5 represents the 2D surface defined by the $K_{1}$ and $K_{2}$ shape parameters. a)
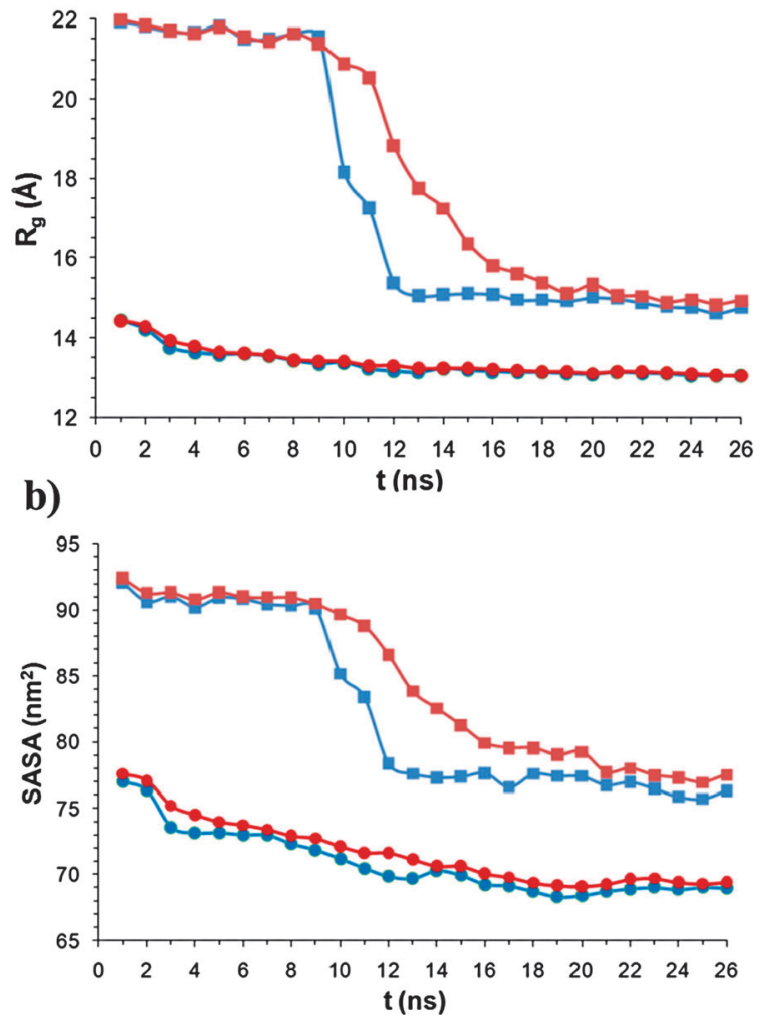

Fig. 3 Evolution of folding indicators during REMD simulations of models III (20-EOPT all-ag $_{2}$ and IV (20-EOPT all-sg $_{2}$ : (a) $R_{\mathrm{g}}$, radius of gyration; (b) SASA, solvent-accessible surface area. The squares indicate simulations beginning from an extended aggregate (model III), and circles indicate simulations beginning from a helical face-to-face aggregate (model IV). Data points are time averages over $1 \mathrm{~ns}$ at temperatures of 281.9 K (blue line) and $300 \mathrm{~K}$ (brown line).

The contour map shown in Fig. 4 indicates the energetic stability based on $R_{\mathrm{g}}$ values. For model III two different maxima were observed. The first one has a $R_{\mathrm{g}}$ close to the initial rod conformation $\left(R_{\mathrm{g}}=21.1 \AA\right)$ while the second one, which appears at the end of the simulation (i.e. it results from the temporal evolution of the initial arrangement), corresponds to the most populated one with $R_{\mathrm{g}}=14.3 \AA$ (Fig. $4 \mathrm{a}$ and b). Moreover, model IV presents a small maximum at $R_{\mathrm{g}}=14.3 \AA$, which corresponds to the initial arrangement, that moves towards a large maximum $\left(R_{\mathrm{g}}=13.1 \AA\right)$, accompanied by a small decrease in energetics (Fig. $4 \mathrm{c}$ and d). In fact, the extended conformation of model III undergoes a very important change in the aggregate shape, its $R_{\mathrm{g}}$ values becoming close to those of the helical shape (Fig. $4 \mathrm{c}$ and $\mathrm{d}$ ).

Analysis of shape parameters on the aggregates, $K_{1}$ and $K_{2}$, which are based on the three principal moments of inertia of the gyration tensor, allows us to determine the evolution of polymers shape. Thus, the shapes of aggregates are determined by the values of $K_{1}$ and $K_{2}$ parameters: an aggregate with $K_{1}$ and $K_{2}$ values tending to 1 corresponds to a spherical shape, a rod shape is obtained when $K_{1}$ and $K_{2}$ tend to 0 and 1 , respectively, and eventually a circular disk or ring is defined when both $K_{1}$ and $K_{2}$ are close to 0.5 . Any other combination tends to intermediate shapes between those being mentioned. 
a)

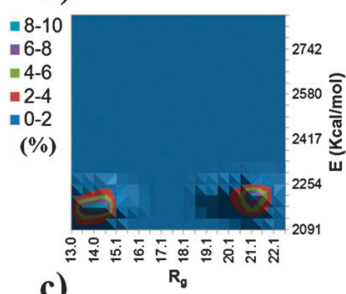

c)

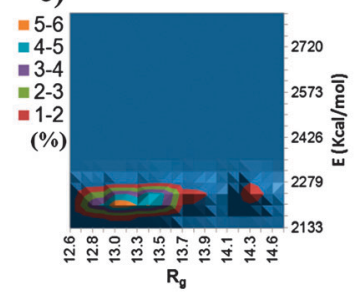

b)

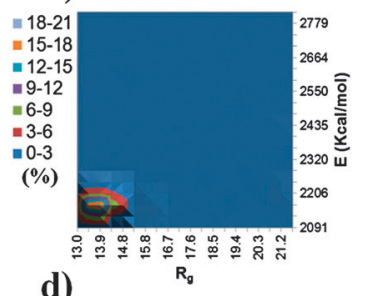

d)

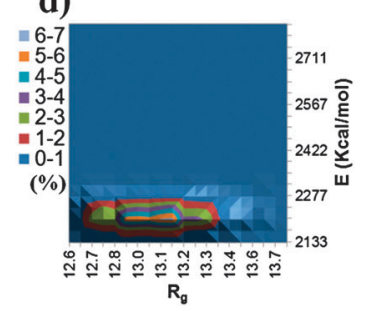

Fig. 4 Contour map of the population (in \%) of aggregates in the 2D space defined by the radius of gyration $\left(R_{\mathrm{g}}\right.$, in $\AA$ ) and the potential energy $\left(E, \mathrm{kcal} \mathrm{mol}^{-1}\right)$ of model III, (a) and (b), and model IV, (c) and (d). Maps displayed in (a) and (c) correspond to the whole REMD simulations while maps in (b) and (d) refer to the last 4 ns of REMD simulations. All data were extracted at $300 \mathrm{~K}$.

Fig. 5a, c and d display the accumulation of populations for the different structures (models III and IV, respectively) along the 26 ns of simulation. An absolute maximum of population at $\left(K_{1}, K_{2}\right)=(0.47,0.79)$, which indicates the predominance of the flat toroid shape, when simulation starts from an extended arrangement (Fig. 5a) is shown, whereas an absolute maximum at $\left(K_{1}, K_{2}\right)=(0.62,0.67)$ is obtained on the model IV evidencing the preservation of the initial helical shape (Fig. 5c).

Noteworthy is the study in detail of the last $4 \mathrm{~ns}$ of simulation on both models, III (Fig. $4 \mathrm{~b}$ and $\mathrm{d}$ ) and IV (Fig. $5 b$ and d). The contour maps of model III show an

a)

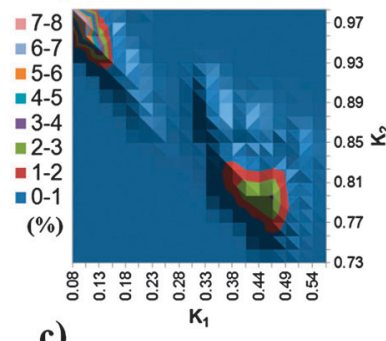

c)

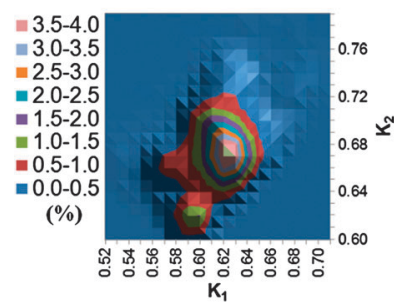

d)
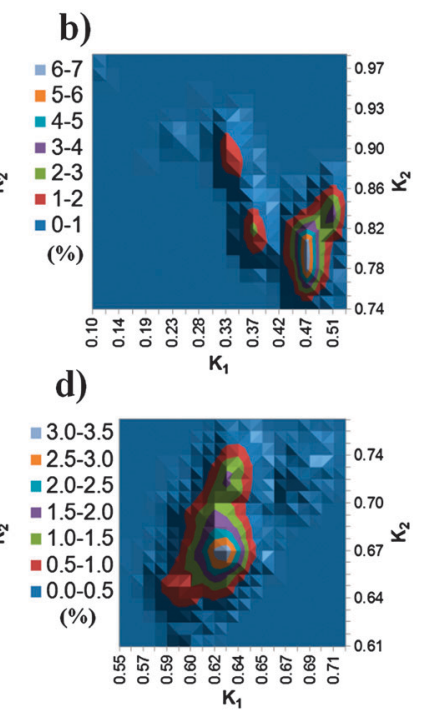

Fig. 5 Contour map of the population (in \%) of aggregates in the 2D space defined by the shape parameters $K_{1}$ and $K_{2}$ of model III, (a) and (b), and model IV, (c) and (d). Maps displayed in (a) and (c) correspond to the whole REMD simulations while maps in (b) and (d) refer to the last 4 ns of REMD simulations. All data were extracted at $300 \mathrm{~K}$. absolute maximum (with $R_{\mathrm{g}}=13.9 \AA$ and $13.2 \AA$, respectively, Fig. $4 \mathrm{~b}$ and d), the potential energy being similar in both cases. Amazingly, the unique maximum found in the $\left(R_{\mathrm{g}}, E\right)$ contour map of model III (Fig. 4b) transforms into four local maxima in the $\left(K_{1}, K_{2}\right)$ contour map (Fig. 5b). Similarly, the unique population maximum shown in the $\left(R_{\mathrm{g}}, E\right)$ contour map of model IV (Fig. 4d) divides into two at the $\left(K_{1}, K_{2}\right)$ surface (Fig. 5d). Thus, the initial extended structure of model III undergoes a strong shape compaction during the simulation, which results in the appearance of several local maxima. Although some of them present an extended structure, the absolute maximum is obtained at a slightly distorted disk-shaped region. On the other hand, the helical conformation of model IV does not retain the initial shape parameters in a narrow region $\left(K_{1}, K_{2}\right)=(0.62,0.67)$, that corresponds to a compact cylindrical shape.

Fig. 6 and Table 4 display the structural characteristics of the most populated arrangements averaged during the last $4 \mathrm{~ns}$ of simulation. For this purpose, a clustering methodology, which allows identification of the most representative structures through the distribution of the RMSD, has been used. As the previous study of maxima located in the $\left(K_{1}, K_{2}\right)$ contour maps reflected a limited number of local maxima, a structural population division made of only five clusters is enough to describe the most representative structural groups.

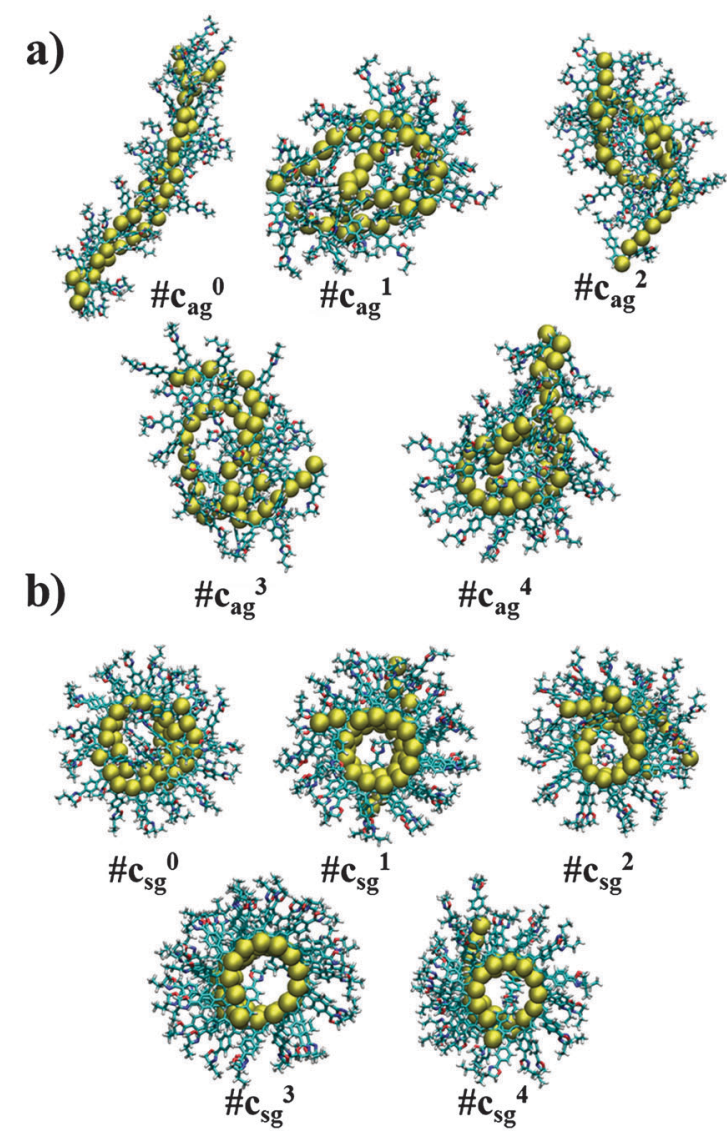

Fig. 6 Representative cluster conformations at $300 \mathrm{~K}$ during the last 4 ns of REMD simulation. Initial conformations were taken from models III (a) and IV (b). The sulfur atom from the polymer backbone has been highlighted to facilitate viewing of the shape. 
Table 4 Summary of the different structures derived from the clustering analysis of the last $4 \mathrm{~ns}$ of REMD simulation at $300 \mathrm{~K}$. Cluster population structures (in \%), radius of gyration $\left(R_{\mathrm{g}}\right.$, in $\AA$ ), and shape parameters $\left(K_{1}\right.$ and $\left.K_{2}\right)$ are shown

\begin{tabular}{lcccc}
\hline \#Cluster & Population & $R_{\mathrm{g}}$ & $K_{1}$ & $K_{2}$ \\
\hline Model III & & & & \\
0 & 3.0 & 21.5 & 0.11 & 0.99 \\
1 & 49.0 & 14.1 & 0.48 & 0.81 \\
2 & 3.5 & 14.5 & 0.41 & 0.82 \\
3 & 1.1 & 14.8 & 0.41 & 0.81 \\
4 & 46.1 & 14.0 & 0.47 & 0.80 \\
Model IV & & & & \\
0 & 2.0 & 13.2 & 0.62 & 0.62 \\
1 & 4.1 & 12.8 & 0.67 & 0.70 \\
2 & 12.0 & 13.0 & 0.63 & 0.68 \\
3 & 57.5 & 13.1 & 0.62 & 0.67 \\
4 & 24.4 & 13.2 & 0.62 & 0.69 \\
\hline
\end{tabular}

Fig. 6 shows the five more representative averaged structures of model III derived from the last 4 ns of simulation, which have been labelled as $\mathrm{c}_{a g}^{i}$ (where $i$ refers to the averaged cluster structures 0 to 4). As seen from Table 4, the structural group $\mathrm{c}_{a g}^{0}$ presents an extended arrangement by merging two helical chains, being stabilized by hiding non-polar interactions from the external polar environment (Fig. 7a). However, the more populated structures correspond to $\mathrm{c}_{a g}^{1}$ and $\mathrm{c}_{a g}^{4}$ (i.e. populations of 49 and $46 \%$, respectively), which show the most compact folding. These structures are obtained by burying the non-polar groups of $\mathrm{c}_{a g}^{0}$, allowing to get a higher stability (Fig. 7b). However, at higher temperatures, representative averaged structures similar to $\mathrm{c}_{a g}^{0}$ become more populated.

Furthermore, five of the most representative averaged structures, derived from those simulations starting from model IV (represented as $\mathrm{c}_{a g}^{i}$, where $i$ refers to the averaged cluster structures 0 to 4 ), show a quite different behaviour with respect to those derived from simulations of model II (Fig. 6 and Table 4). In this case no significant changes were observed, only an apparent reduction in the helix diameter of roughly $2 \AA$ in the most populated cluster structure, $\mathrm{c}_{s g}^{3}$, which presents an ellipsoidal helix. Interestingly, the conformations with less population correspond to those with a partial unscrew at the end of the helix and, therefore, with higher conformational energy.

Contour maps and clustering analysis from model III indicate an important feature related to the aggregates conformation: the coexistence of extended and globular shapes that could be

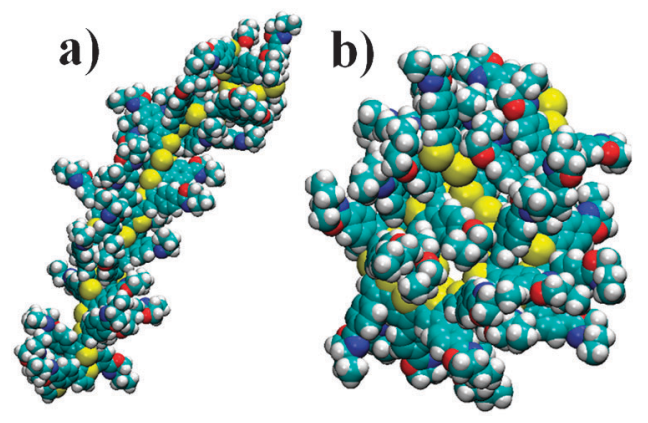

Fig. 7 Details of side chain arrangement and burying of non-polar moieties on the representatives cluster conformations (a) $\mathrm{c}_{a g}^{0}$ and (b) $\mathrm{C}_{a g}^{1}$.

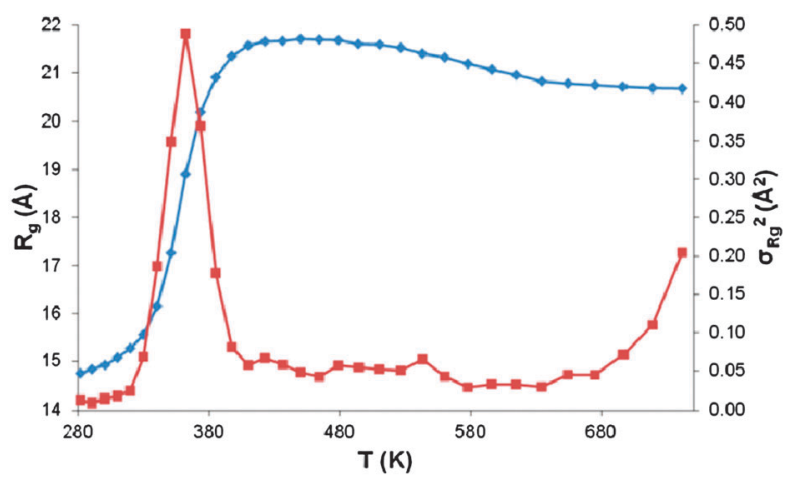

Fig. 8 Temperature dependences of the radius of gyration $R_{\mathrm{g}}$ of model III (blue diamonds) and its mean-square fluctuations $\sigma_{R_{\mathrm{g}}}^{2}$ (brown squares).

attributed to the transition between the coil and the globular shape. In order to characterize this coil-globule transition, the averaged $R_{\mathrm{g}}$ during the last $4 \mathrm{~ns}$ was considered and its temperature evolution was plotted (see Fig. 8). $R_{\mathrm{g}}$ of all involved conformations and its mean-square fluctuations $\left(\sigma_{R_{\mathrm{g}}}^{2}=\left\langle R_{\mathrm{g}}^{2}\right\rangle-\left\langle R_{\mathrm{g}}\right\rangle^{2}\right)$ were shown to be useful parameters for the characterization of the phase transition of flexible polymers and peptides in solution. ${ }^{35-38}$ Fig. 8 presents a sigmoid like increase with the temperature, indicating a transition from the state containing the compact polymer aggregate $\left(R_{\mathrm{g}} \approx 14.9 \AA\right.$, at $300 \mathrm{~K})$ to a more extended state $\left(R_{\mathrm{g}} \approx 21.7 \AA\right.$, at $\left.436 \mathrm{~K}\right)$. The sigmoid function and its mean-square fluctuations $\sigma_{R_{\mathrm{g}}}^{2}$ indicate the midpoint of the transition at $T \approx 362 \mathrm{~K}$. This clear sigmoid function was not observed when the averaged $R_{\mathrm{g}}$ from those simulations using model IV as a starting point was plotted against temperature (not shown). The structural forms before and after the coil-globule transition are associated with the clusters $\mathrm{c}_{a g}^{0}$ and $\mathrm{c}_{a g}^{1}$ (Fig. 6) derived from the clustering analysis of model III REMD trajectory, at $300 \mathrm{~K}$. This reinforces the hypothesis that the extended double helical chain is more stable at higher temperatures than at the coil-globule transition temperature $(362 \mathrm{~K})$, while the folded shape of the double helix appears at lower temperatures.

The data obtained from this theoretical study agree with the available experimental data, which point to the formation of $\pi$-stacked chiral aggregates. ${ }^{10,15,17}$ However, such stacking becomes less rigid for relatively long chains in solution due to the flexibility of PEOPT chains. However, interactions between thiophene rings and between aromatic side groups allow the stabilization of a pseudo-helical structure that could enhance the chirality of this polymer in poor solvents.

\section{Octamer's aggregate}

The singular behaviour of 8-EOPT with respect to other oligomers with a higher number of repeating units at aggregation time deserves special attention. Sakurai et al. ${ }^{16}$ evidenced that the octamer presents an isosbestic point and no change in the ICD, independently of the solvent. In contrast, similar ICDs with solvatochromism have been observed for larger oligomers, even though the Cotton effect signs were found to be significantly dependent on the solvent. In our previous work we reported that 8-EOPT prefers the $\mathrm{sg}^{+} \mathrm{sg}^{-}$ring conformation with respect to 

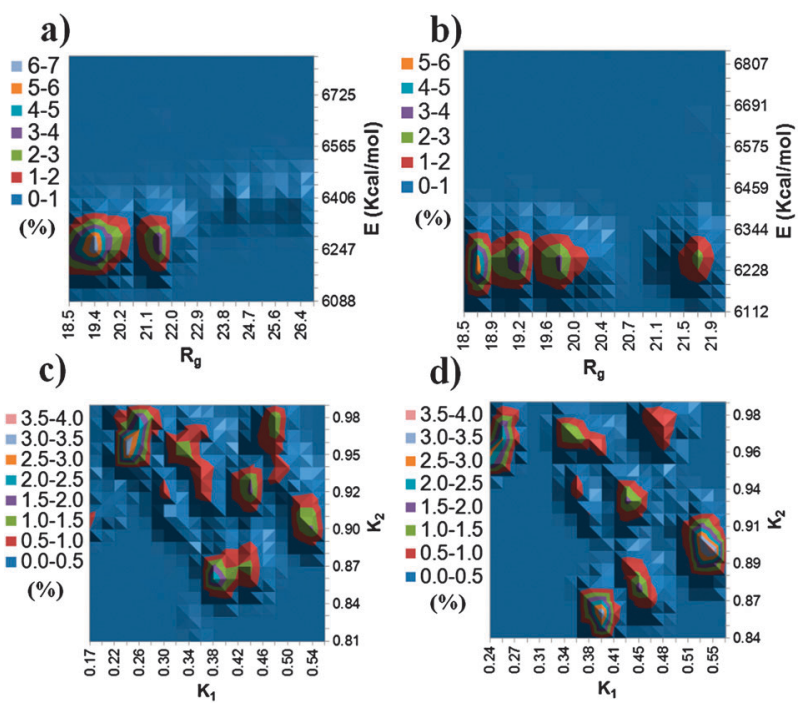

Fig. 9 Contour map of the population (in \%) of $\left(8-\mathrm{EOPT}_{s g^{+}} s g^{-}\right)_{16}$ (model $\mathrm{V}$ ) aggregates in the $2 \mathrm{D}$ space defined by the radius of gyration ( $R_{\mathrm{g}}$, in $\AA$ ) and the potential energy $\left(E, \mathrm{kcal} \mathrm{mol}^{-1}\right)$ during: (a) the whole REMD simulation; and (b) the last 4 ns of REMD simulation. Contour map of the population (in \%) of the same aggregates in the 2D space defined by the shape parameters $K_{1}$ and $K_{2}$ during: (c) the whole REMD simulation; and (d) the last 4 ns of REMD simulation. All trajectories conformation was extracted at $300 \mathrm{~K}$.

the all- $\mathrm{sg}^{+}$helix conformation in both the gas phase and acetonitrile. ${ }^{18}$ So, this stiffer and flat octamer of semicircular shape could influence the formation of chiral supramolecular aggregates because its rigid and flat structure can easily form new intermolecular interactions. In order to investigate the influence of the structure on the aggregation, a simulation was carried out using model $\mathrm{V}$ as a starting point (i.e. 16 8-EOPT molecules $\pi$-stacked along their backbone, as indicated in Table 1). The simulation was performed using the REMD methodology and considering sixteen different temperatures during 20 ns.

Fig. 9 plots contour maps of the conformational population against $R_{\mathrm{g}}$ and the potential energy on one hand (Fig. 9a and b), and shape parameters such as $K_{1}$ and $K_{2}$ (Fig. 9c and d) on the other. Comparison of long and short simulation trajectory graphs evidences that the most favoured conformations are those with $R_{\mathrm{g}}=19.4 \AA$. Regarding structural parameters, three main conformational ensembles were stabilized, their population maxima of $3.8,3.6$, and $2.7 \%$ being located at the shape parameters of $\left(K_{1}, K_{2}\right)=(0.55,0.90),(0.39,0.86)$, and $(0.24,0.96)$, respectively. Thus, the initial aggregate, which holds a rod shape made of $\pi$-stacked octamers (model V) with conformational parameters $R_{\mathrm{g}}=25.7 \AA$ and $\left(K_{1}, K_{2}\right)=$ $(0.19,0.89)$, keeps an elongated shape because of its large values of $K_{2}$. However, the significant increase in $K_{1}$ in the most populated structures reflects an increase in the radial inertial mass, indicating a major reorganization in this direction.

Fig. 10 and Table 5 present the characteristics of the most populated structures averaged during the last $4 \mathrm{~ns}$ of simulation at $300 \mathrm{~K}$, which were obtained using the clustering methodology. The most populated structure at $300 \mathrm{~K}$ $\left(\mathrm{c}_{s g^{+} s g^{-}}^{4}\right)$ corresponds to a new twisted structure around the

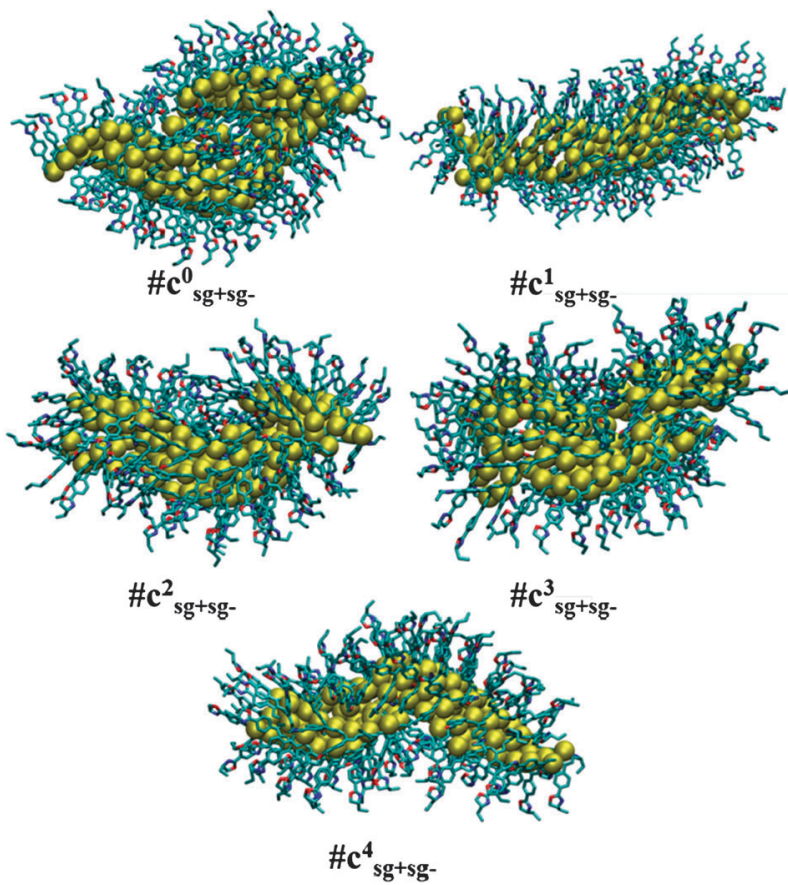

Fig. 10 Representative structures for each group derived from the cluster analysis. The data were derived during the last 4 ns of REMD simulation at $300 \mathrm{~K}$, the $\left(8-\mathrm{EOPT}_{s g^{+}} s g^{-}\right)_{16}$ (model V) being the starting arrangement. In order to facilitate viewing of the shape, the sulfur atoms of the polymer backbone have been highlighted while the hydrogen atoms have been hidden.

Table 5 Summary of the different structures derived from the clustering analysis of the last $4 \mathrm{~ns}$ of REMD simulation using $\left(8-\mathrm{EOPT}_{s g+} s g-\right)_{16}$ (model V) aggregate as starting arrangement, at $300 \mathrm{~K}$. Cluster population structures (in \%), radius of gyration $\left(R_{\mathrm{g}}\right.$, in $\AA$ ), and shape parameters $\left(K_{1}\right.$ and $\left.K_{2}\right)$ are shown

\begin{tabular}{lllll}
\hline \#Cluster & Population & $R_{\mathrm{g}}$ & $K_{1}$ & $K_{2}$ \\
\hline 0 & 32.9 & 19.0 & 0.52 & 0.92 \\
1 & 18.2 & 21.4 & 0.26 & 0.98 \\
2 & 5.6 & 19.3 & 0.44 & 0.94 \\
3 & 0.8 & 19.3 & 0.46 & 0.88 \\
4 & 42.5 & 19.4 & 0.43 & 0.89 \\
\hline
\end{tabular}

rod axis of the initial starting point followed by a tiny shift along the direction of the rod axis, which allows us to avoid a large helical radius. This structure, which appears to be broken at some points, presents a kind of chain hooks as building blocks forming a self-assembled supramolecular structure. These building blocks are made of $6-8 \pi$-stacked octamers, each joining together along the rod axis. The less populated conformations at this temperature are those with chain hooks made of less octamer units (i.e. 3-4 $\pi$-stacked octamers forming an helix with a smaller axial diameter, $\left.\mathrm{c}_{s g^{+}}^{1} s g^{-}\right)$, and those with a more dispersed helical shape $\left(\mathrm{c}_{s g^{+} s g^{-}}^{2}\right.$ and $\left.\mathrm{c}_{s g^{+} s g^{-}}^{3}\right)$, where the union of these chain hooks is not showing a clear helical structure.

Similarly to the previous oligomer aggregation section, a coil-globule transition has been studied on model V. Therefore, the representative structure of the most populated cluster during the last 4 ns of REMD trajectory at each temperature has been obtained by using cluster analysis, the structural 


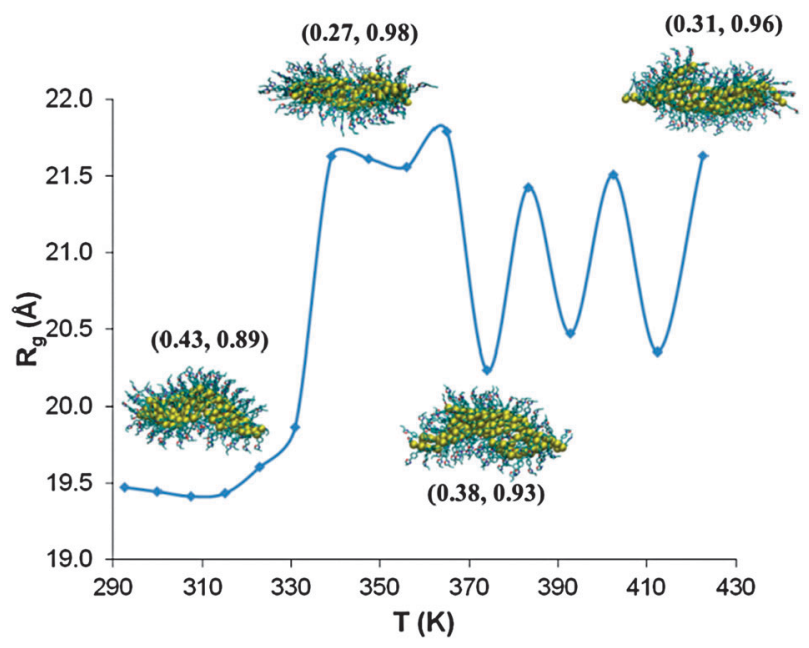

Fig. 11 Temperature dependence of the radius of gyration $R_{\mathrm{g}}$ for the most populated representatives derived from the cluster analysis using the last $4 \mathrm{~ns}$ of REMD simulation. The (8-EOPT $\left.g^{+}{ }^{+} g^{-}\right)_{16}($ model V) was the starting arrangement and the shape parameters $K_{1}$ and $K_{2}$ are displayed in parentheses.

parameters evolution being plotted against temperature in Fig. 11. Interestingly, $R_{\mathrm{g}}$ of the most populated structures shows a near-sigmoidal transition temperature at around $335 \mathrm{~K}$, where the aggregate structure has an important conformational change going from a helical chain with hook rings made of 6 to 8 octamer units $\left(\mathrm{c}_{s g^{+} s g^{-}}^{4}\right.$ in Fig. 10) to another helical chain with hook rings made of less octamer units $\left(\mathrm{c}_{s g^{+} s g^{-}}^{1}\right.$ in Fig. 10). Moreover, the helix instabilities are reflected beyond $360 \mathrm{~K}$ with an oscillating behaviour of $R_{\mathrm{g}}$, which correspond to a decrease in stacked octamer units and a dispersion of the helical structure led by the entropic component of the global system.

\section{Conclusions}

In this work, MD and REMD simulations have been used to carry out a systematic study on the conformational preference of PEOPT and some of its simple chain aggregates. First, MD calculations using explicit solvent molecules of growing polarity have been used to explore the conformation of a single PEOPT chain starting from two independent extended and helical conformations. Results allow us to conclude that there is a clear trend towards stabilization of the syn-gauche conformation with respect to the anti-gauche conformation in the system. This tendency can be attributed to the stress induced on the polymer backbone by the side chain interactions. An increase in solvent polarity induces a narrowing of the end-to-end distance, as well as an enhancement of the interactions between the side groups, specifically those which have a higher percentage of syn-gauche rotamers between consecutive thiophene rings. Indeed, the folded structure observed along the simulation is due to the formation of bent structures of the polymer rather than a helical conformation.

Secondly, two REMD simulations of a PEOPT aggregate made of two single chains were conducted, one was started considering an extended conformation while in the other molecules adopt a helical arrangement. Our simulation results lead to conservation of globular structure, showing a predominance of a flat toroid shape when simulation starts from an extended conformation, whereas the helical arrangement is preserved when this motif is used as a starting point. Clustering analysis of the final trajectory shows that at high temperatures the aggregate presents an extended conformation by merging two molecules in a helicoidal shape, being stabilized by hiding non-polar interactions. At low temperatures, the previous helical disposition is folded on itself, burying non-polar groups to get a better stabilization. The extended aggregate presents a clear coil-globule transition at $362 \mathrm{~K}$, between the extended double helical chain stabilized at higher temperatures and the folded shape of the double helix found at lower temperatures.

Finally, a REMD simulation of a set of octamer aggregates has been performed. The shape of the most stable octamer conformation has been shown as an important point in the formation of chiral supramolecular aggregates because its rigid, flat, and semicircular structure allows stacking. Our results show a self-assembled supramolecular structure made of hook rings as building blocks (a set of stacked octamers) that are cross-linked to form a pseudo-helical structure. Non-polar moieties are buried within the heart of this pseudo-helix. Temperature dependence was observed at around $335 \mathrm{~K}$, when the supramolecular structure shifts from a helical chain with hook rings made of 6 to 8 octamer units to another with fewer octamer units on the hook rings.

PEOPT appears to be less rigid than previously thought, and its behaviour in solution is flexible enough to show a coil appearance. On the other hand a strong tendency to aggregation was observed in poor solvent, stabilizing structures that reinforce the chirality of the polymer, in concordance with experimental data. Two different aggregations have been observed depending on the oligomer length, a short and rigid oligomer induces the formation of a self-assembled helical aggregate by a bottom-up stacking, whereas larger oligomers tend to form an aggregation of bent double helices.

\section{Acknowledgements}

This work has been supported by MICINN and FEDER (Grant MAT2009-09138), and by the Generalitat de Catalunya (research group 2009 SGR 925 and XRQTC). The authors are indebted to the Barcelona Supercomputer Center (BSC) for the computational resources provided. Support for the research of C.A. was received through the prize "ICREA Academia" for excellence in research funded by the Generalitat de Catalunya.

\section{Notes and references}

1 A. O. Patil, A. J. Heeger and F. Wudl, Chem. Rev., 1988, 88, 183-200.

2 J. Roncali, Chem. Rev., 1992, 92, 711-738.

3 J. Roncali, Chem. Rev., 1997, 97, 173-206.

4 E. Yashima, H. Goto and Y. Okamoto, Macromolecules, 1999, 32, $7942-7945$.

5 H. Goto, E. Yashima and Y. Okamoto, Chirality, 2000, 12, 396-399.

6 M. M. Bouman, E. E. Havinga, R. A. J. Janssen and E. W. Meijer, Mol. Cryst. Liq. Cryst., 1994, 256, 439-448.

7 L. Pu, Acta Polym., 1997, 48, 116-141. 
8 T. Yamamoto, D. Komarudin, M. Arai, B.-L. Lee, H. Suganuma, N. Asakawa, Y. Inoue, K. Kubota, S. Sasaki, T. Fukuda and H. Matsuda, J. Am. Chem. Soc., 1998, 120, 2047-2058.

9 B. M. W. Langeveld-Voss, R. A. J. Janssen and E. W. Meijer, J. Mol. Struct., 2000, 521, 285-301.

10 H. Goto, Y. Okamoto and E. Yashima, Macromolecules, 2002, 35, 4590-4601.

11 P. V. Shibaev, K. Schaumburg, T. Bjornholm and K. Norgaard, Synth. Met., 1998, 97, 97-104.

12 P. V. Shibaev and K. Schaumburg, Synth. Met., 2001, 124, 291-294.

13 N. Kiriy, E. Jaehne, H.-J. Adler, M. Schneider, A. Kiriy, G. Gorodyska, S. Minko, D. Jehnichen, P. Simon, A. A. Fokin and M. Stamm, Nano Lett., 2003, 3, 707-712.

14 Y. D. Park, H. S. Lee, Y. J. Choi, D. Kwak, J. H. Cho, S. Lee and K. Cho, Adv. Funct. Mater., 2009, 19, 1200-1206.

15 H. Goto, Y. Okamoto and E. Yashima, Chem.-Eur. J., 2002, 8, 4027-4036.

16 S.-i. Sakurai, H. Goto and E. Yashima, Org. Lett., 2001, 3, 2379-2382.

17 H. Goto and E. Yashima, J. Am. Chem. Soc., 2002, 124, $7943-7949$.

18 O. Bertran, J. Torras and C. Alemán, J. Phys. Chem. C, 2010, 114, 11074-11080.

19 Y. Sugita and Y. Okamoto, Chem. Phys. Lett., 1999, 314, 141-151.

20 A. Mitsutake, Y. Sugita and Y. Okamoto, Pept. Sci., 2001, 60, 96-123.

21 J. Preat, F. Rodríguez-Ropero, J. Torras, O. Bertran, D. Zanuy and C. Alemán, J. Comput. Chem., 2010, 31, 1741-1751.

22 J. Wang, R. M. Wolf, J. W. Caldwell, P. A. Kollman and D. A. Case, J. Comput. Chem., 2004, 25, 1157-1174.

23 D. A. Case, T. A. Darden, I. T. E. Cheatham, C. L. Simmerling, J. Wang, R. E. Duke, R. Luo, R. C. Walker, W. Zhang, K. M. Merz, B. Roberts, B. Wang, S. Hayik, A. Roitberg,
G. Seabra, I. Kolossváry, K. F. Wong, F. Paesani, J. Vanicek, X. Wu, S. R. Brozell, T. Steinbrecher, H. Gohlke, Q. Cai, X. Ye, J. Wang, M.-J. Hsieh, G. Cui, D. R. Roe, D. H. Mathews, M. G. Seetin, C. Sagui, V. Babin, T. Luchko, S. Gusarov, A. Kovalenko and P. A. Kollman, AMBER 11, University of California, San Francisco, 2010.

24 P. Cieplak, J. Caldwell and P. Kollman, J. Comput. Chem., 2001, 22, 1048-1057.

25 X. Grabuleda, C. Jaime and P. A. Kollman, J. Comput. Chem., 2000, 21, 901-908.

26 J.-P. Ryckaert, G. Ciccotti and H. J. C. Berendsen, J. Comput. Phys., 1977, 23, 327-341.

27 T. Darden, D. York and L. Pedersen, J. Chem. Phys., 1993, 98, 10089-10092.

28 G. D. Hawkins, C. J. Cramer and D. G. Truhlar, J. Phys. Chem., 1996, 100, 19824-19839.

29 M. L. Connolly, Science, 1983, 221, 709-713.

30 S. Sridharan, A. Nicholls and K. A. Sharp, J. Comput. Chem., 1995, 16, 1038-1044.

31 P. G. Khalatur, Polym. Sci. USSR, 1980, 22, 2438-2448.

32 V. A. Ivanov, M. R. Stukan, V. V. Vasilevskaya, W. Paul and K. Binder, Macromol. Theory Simul., 2000, 9, 488-499.

33 J. Shao, S. W. Tanner, N. Thompson and T. E. Cheatham, J. Chem. Theory Comput., 2007, 3, 2312-2334.

34 O. Bertran, J. Torras and C. Alemán, J. Phys. Chem. C, 2010, 114, 11074-11080.

35 V. A. Ivanov, W. Paul and K. Binder, J. Chem. Phys., 1998, 109, 5659-5669.

36 M. Cecchini, F. Rao, M. Seeber and A. Caflisch, J. Chem. Phys., 2004, 121, 10748-10756.

37 G. Singh, I. Brovchenko, A. Oleinikova and R. Winter, J. Phys. Chem. B, 2009, 113, 9863-9870.

38 T. Li, X. Yang and E. Nies, J. Chem. Theory Comput., 2010, 7, 188-202. 